\title{
EL ESPAÑOL Y EL LESCO EN EL MARCO DE LA ENSEÑANZA DE UNA SEGUNDA LENGUA PARA LAS PERSONAS SORDAS EN COSTA RICA ${ }^{1}$
}

\author{
Carlos Sánchez Avendaño
}

Recibido 30-VIII-2004 • Aceptado 7-IX-2004

\begin{abstract}
Resumen: Este ensayo se dedica a explorar algunos problemas concomitantes a la nueva concepción educativa de la persona sorda en Costa Rica; entre ellos, el lugar del LESCO dentro del currículum, la idea de que el español constituye una segunda lengua que debe enseñarse en su registro escrito, el valor que nuestra sociedad le otorga a lo oral y sus implicaciones en el nuevo modelo pedagógico, la alfabetización de los sordos, la formación de los docentes, y el indispensable cambio de actitud hacia la persona sorda $y$ el LESCO que debe suscitarse en el país.
\end{abstract}

Palabras clave: Educación de la persona sorda, segundas lenguas, educación bilingüe, LESCO, alfabetización de la persona sorda.

\begin{abstract}
This essay explores some concomitant problems of the new educational conception of deaf people in Costa Rica. Among these problems are: the place of LESCO in the curriculum, the idea of teaching Spanish as a second language writing, the value society gives to the oral component and its implication in this conception. The article also examines topics such as teacher training and the change of attitude required for deaf people and LESCO in Costa Rica.
\end{abstract}

Key words: Education for the deaf, second language writing, bilingual education, LESCO, literacy in deaf people.

\section{Introducción}

En esta reflexión me ocuparé de un tema que parece perfilarse como de especial relevancia en la educación de las personas sordas, tanto en Costa Rica como en el resto del mundo. Frente a los diversos modelos con que se ha abordado la escolarización de las personas en condición de pérdida auditiva (la oralización y la comunicación total, por mencionar los dos grandes paradigmas, aún vigentes en muchos casos), en la actualidad se propone un modelo de educación bilingüe, en el que el español dejaría, por consiguiente, de entenderse y enseñarse como lengua materna para ser tratado como segunda lengua.

Este cambio de concepción, en apariencia simple, no es ni mucho menos un mero artificio nominal, aunque la dificultad que conlleva y la falta de capacitación de los docentes y de recursos adecuados muchas veces provoque que, en la práctica, no se verifique la enseñanza del español como una segunda lengua, con todo lo que ello implica: ¿cuál variedad de español debe enseñarse?, ¿cuál modalidad -oral o escrita- debe privilegiarse y con cuáles argumentos?, ¿qué implica enseñar la modalidad escrita?, ¿cuál es el mejor método?, y, sobre todo, ¿comporta la enseñanza de una segunda lengua oral a una persona sorda 
una especificidad nunca contemplada en la teoría sobre la adquisición de segundas lenguas?

Además, de inmediato también surge la gran incógnita sobre el papel que debe ocupar el LESCO (lengua de señas costarricense) en dicho modelo bilingüe: ¿debe ser lengua auxiliar de instrucción, a la que se recurrirá únicamente como vehículo para iniciar el proceso de adquisición del español?, ¿debe ocupar un lugar en el currículum?, ¿cuál es -a fin de cuentas- el modelo de educación bilingüe que más conviene?

Las opiniones que voy a exponer a continuación deben interpretarse como una reflexión desde mi formación de lingüista. En este sentido, se trata de una visión de observador externo: no soy ni miembro de la comunidad sorda, directamente afectada por las decisiones que se tomen sobre su educación desde las esferas de poder, ni tampoco soy docente de sordos. Soy un lingüista interesado en la educación idiomática, quien se ha visto, por diversas circunstancias, relacionado con el tema de la enseñanza del español a las personas sordas. De los cortos espacios en los que he podido compartir -y sobre todo aprendercon sordos y, en particular, con los docentes encargados de su instrucción, surgen estas reflexiones.

\section{La lengua materna o primera y la segunda lengua}

Cuando nos situamos en el marco de la enseñanza de una segunda lengua, necesariamente debemos hacer alusión a la existencia de una primera lengua, comúnmente denominada lengua materna. He aquí donde aparece el primer gran problema en la educación del sordo.

La primera lengua es aquella lengua particular que se desarrolla en el proceso de adquisición del lenguaje; esto es, el proceso en el cual nuestra capacidad lingüística se verifica y empezamos a comprender y producir emisiones en una lengua concreta. Si bien todo apunta a que adquirir una lengua forma parte de una facultad innata en el ser humano, también se reconoce que dicha facultad resulta insuficiente sin la interacción con adultos que la empleen con el niño. En términos técnicos, se necesita de un input en una lengua concreta para que se detone el proceso de adquisición del lenguaje. Así, la capacidad lingüística sería una facultad genética, pero la adquisión de una lengua particular depende de la transmisión cultural.

A riesgo de simplificar demasiado, los puntos centrales que se desprenden de las diversas teorías de la adquisición del lenguaje se pueden resumir en los siguientes (Berko y Bernstein, 1999):

1. Los principios del lenguaje son innatos, no aprendidos. Así, no se cree que exista alguna lengua particular transmitida genéticamente, sino que se trata de un bioprograma que se desarrolla cuando el niño se ve expuesto a una lengua (teoría innatista).

2. El lenguaje es un aspecto de la cognición humana. De esta forma, la adquisición del lenguaje es concomitante al desarrollo cognitivo en el niño o, incluso, está subordinado a este (teoría cognitiva).

3. El lenguaje solamente se puede desarrollar en la interacción comunicativa con otros seres humanos. La mera exposición a una lengua concreta no es suficiente, sino que se necesita adquirir la lengua en función de las necesidades comunicativas en situaciones reales (teoría de la interacción social).

A estas teorías, habría que sumar una hipótesis que vendría a relativizar lo de la capacidad innata para adquirir el lenguaje en todos los seres humanos. Según la hipótesis del período crítico o sensible, dicha capacidad puede desarrollarse sin dificultades antes de la pubertad (alrededor de los 11 o 12 años); de lo contrario, el 
proceso de adquisición del lenguaje puede verse sumamente afectado. La existencia de este período crítico es aún una cuestión debatida, pero se han hallado pruebas en los casos de los llamados "niños salvajes"; es decir, en los niños que han crecido aislados de otros seres humanos o que han recibido un estímulo lingüístico y social muy pobre: estos niños enfrentan grandes dificultades para desarrollar su capacidad comunicativa en una lengua adulta. Por consiguiente, es probable que, en efecto, exista un período en el cual el lenguaje se adquiera con mayor facilidad, entre otros factores por la mayor plasticidad neuronal durante la infancia y la maduración aún en proceso de los órganos motores (Fernández, 1999).

A partir de todas las consideraciones anteriores, la primera lengua sería la que acompaña el proceso de adquisición del lenguaje; esto es, la lengua en la que se hacen patentes los principios innatos que poseemos como especie humana, la lengua que se manifiesta en forma paralela a nuestro desarrollo cognitivo general, y la lengua en la que entablamos nuestras primeras interacciones sociales; es, además, la lengua que -idealmente- adquirimos dentro de los límites del período crítico ${ }^{2}$.

Por su parte, una segunda lengua es todo idioma que se adquiera sobre la base de la existencia de una primera lengua previa. Siguiendo este supuesto se han elaborado muchas de las teorías de la adquisición de una segunda lengua, pues se parte de que, en principio, la mayoría de los seres humanos hablan al menos un idioma con anterioridad (Larsen y Long, 1994):

1. Al adquirir una segunda lengua, las personas pasan necesariamente por el filtro de su primer idioma; en otras palabras, realizan diversos tipos de transferencias de su lengua materna a la lengua que están aprendiendo. Esta transferencia consiste en una estrategia necesaria en los primeros estadios de adquisición (análisis contrastivo y teoría de la transferencia).
2. Poder comunicarse en una lengua es el resultado de la adquisión y no su causa; es decir, no es sino hasta que la adquisición se ha verificado que somos capaces de emplear la lengua. La capacidad innata para el lenguaje sigue disponible para los hablantes en la adquisición de una segunda lengua de forma natural, pero lo que se aprenda conscientemente por medio de la instrucción formal solo sirve para revisar y corregir las emisiones que se producen (teoría del monitor).

3. En los primeros estadios de la adquisición de una segunda lengua, se producen procesos de simplificación análogos a los que hallamos en situaciones de interacción verbal en las cuales los participantes hablan lenguas distintas y deben crear una herramienta de comunicación común sobre la base de sus lenguas (hipótesis de la pidginización).

Así las cosas, se han esbozado varias diferencias entre la adquisición de una primera lengua y un segundo idioma (Vega, 1990; Larsen y Long, 1994), a saber:

a. El aprendizaje de la segunda lengua no va, por lo general, paralelo al desarrollo cognitivo general en el individuo.

b. En la primera lengua, cuando niños, nos comunicamos con emisiones incompletas y palabras cuyo significado es muy amplio; no se espera del niño emisiones perfectas ni acabadas en sus primeros años. Por el contrario, en una segunda lengua, se espera que el aprendiz se comunique con emisiones completas y correctas desde el principio.

c. En el proceso de adquisición de la lengua materna, esta es la única lengua a la que se puede recurrir para comunicarse. Por el contrario, en el caso de las segundas lenguas, las personas recurren al soporte de su primera 
lengua; de ahí, por ejemplo, que traduzcan o que primero elaboren su pensamiento en la lengua materna y luego busquen la forma de expresarlo en el idioma meta.

d. Tampoco existe en la primera lengua un idioma que pueda provocar ningún tipo de interferencia.

e. El proceso de adquisición de la lengua materna se realiza en situaciones normales y cotidianas de interacción social. No ocurre así, por lo general, con las segundas lenguas, las cuales suelen aprenderse en contextos formales de instrucción explícita y programada.

En este marco general, ¿dónde se ubican el español y el LESCO en el caso de la educación de la persona sorda?

En la situación ideal -pero contrafactual- de un niño sordo que, desde sus primeros meses de vida, reciba su estímulo lingüístico en LESCO y luego reciba instrucción para aprender el español, no cabe duda de que estamos ante un ejemplo típico de adquisición de una segunda lengua: el LESCO es la lengua materna o primera lengua de dicho niño, pues es en la que se verificó el bioprograma de adquisición del lenguaje, concomitante a su desarrollo cognoscitivo y al establecimiento de sus primeras interacciones lingüísticas. El español se adquirirá, entonces, sobre esta base primigenia; es decir, será su segunda lengua. No obstante, como bien sabemos, difícilmente nos encontramos con esta situación cuando de los niños sordos se trata.

Lo usual es que el niño sordo no se vea estimulado lingüísticamente en las lenguas cuyas características le permiten potenciar su capacidad para adquirir el lenguaje ante la presencia de pérdida auditiva: las lenguas de señas. Se suele decir, al respecto, que las lenguas de señas son las lenguas naturales para los sordos por cuanto estas se codifican empleando un canal viso-gestual, es decir, que estimulan la recepción visual del mensaje y la producción por medio de diversos articuladores análogos a los del aparato fonador: las manos y las diversas configuraciones que pueden adquirir, la cara y las diferentes expresiones, el manejo del espacio y del cuerpo, etc.

Cualquier lengua oral como el español, o sea, una lengua que se actualiza en un canal acústico-auditivo, resulta una lengua no natural para una persona con una condición de pérdida auditiva, pues el sonido no sirve de estímulo lingüístico en tal caso. Por ende, un niño sordo que únicamente se vea expuesto a emisiones orales no está siendo estimulado para que ponga en marcha su capacidad lingüística innata. El punto central aquí es que el input (el material lingüístico que recibe el aprendiz) constituye el detonante del proceso de adquisición. Si ese input es demasiado pobre o resulta inaccesible, entonces tal proceso no se verificará con éxito. Así, el input oral en español nunca será realmente útil para que un niño con sordera severa adquiera el lenguaje; esto es, en principio ninguna lengua oral podrá ser su lengua materna, en el sentido más estricto y técnico, como el que hemos manejado aquí.

En este punto es en el que se pueden encontrar los mayores problemas en la conceptualización de la enseñanza de una segunda lengua para los sordos. En el caso -lamentablemente atípico, como ya se dijode un niño sordo que pasó por el proceso de adquisición del lenguaje a partir de estímulos e interacción en una lengua de señas, sin duda podrá decirse que el español se concebirá como una segunda lengua, con las particularidades del caso en las que nos detendremos luego. Pero, ¿qué sucede con los niños -una alarmante mayoría, según me lo han expresado repetidas veces los docentes de sordos- que entran a la escuela sin haber recibido ese tipo de input?

Como es de esperar, estos niños no hablan ni español ni LESCO; por lo tanto, de inmediato nos asaltan varias interrogantes: ¿tienen una lengua materna?, ¿qué implicaciones tendrá el retraso en la adquisición del lenguaje en su desarrollo cognitivo 
general?, ¿cómo afecta la falta de una primera lengua efectivamente desarrollada en el aprendizaje del español como segunda lengua?, ¿se puede enseñar una segunda lengua sin desarrollar primero la lengua materna natural de los niños?, ¿los jóvenes que inician su escolarización tardíamente podrán alcanzar una competencia aceptable en español? No pretendo darles respuesta a preguntas de semejante envergadura; sin embargo, considero impostergable su discusión seria y profunda con miras a programar a largo plazo lo que se quiere y puede alcanzar con los modelos educativos que se propongan, en consonancia con la realidad socio-cultural del país. Cito, a este respecto, las palabras de Monserrat Veyrat Rigat:

"Las recientes investigaciones sobre el desarrollo cognitivo y lingüístico de los niños sordos demuestran que los insuficientes resultados académicos que éstos alcanzan en su etapa escolar no se deben tanto a una incapacidad a causa de la sordera como a la escasa exposición a intercambios comunicativos con el resto de la sociedad puesto que no disponen de un código lingüístico común, y en muchas ocasiones, tanto la actitud de los padres como de la escuela, no les facilita la posibilidad de interiorizar un sistema lingüístico desde épocas tempranas con el que poder organizar sus experiencias cotidianas" (Veyrat Rigat 1998, p. 3).

\section{Consideraciones sobre la enseñanza del español como segunda lengua a las personas sordas}

\subsection{La oralidad en nuestra cultura}

Resulta fácil entender por qué una persona sorda debe aprender español en nuestra sociedad: está inmersa en un medio en el que la lengua de la comunicación habitual y oficial es el español. Sin un conocimiento de este, cualquier persona en nuestro país está condenada al aislamiento y la exclusión; sobre todo, si se considera que, en la mayoría de los casos, incluso sus padres y hermanos son hablantes de español y no de LESCO. Asimismo, aprender español en el caso de Costa Rica consiste en un derecho para la persona sorda, a la luz de la Declaración universal de derechos lingüísticos (Artículo 13): “Todo el mundo tiene derecho a acceder al conocimiento de la lengua propia del territorio donde reside" (Comité de seguimiento, 1996, p.19). Lo que no pareciera tan claro es cuál modalidad del español debería aprender.

Por lo general, en la actualidad, la enseñanza de segundas lenguas se ha venido centrando en el registro oral. Por ello mismo, vemos cómo cada día con más insistencia se anuncian cursos conversacionales, los cuales se promocionan como métodos enfocados en la comunicación. Esto evidencia el gran auge de lo oral que nuevamente está experimentando nuestra sociedad: lo importante -se cree-, al aprender una segunda lengua, es hablar y entender lo que se escucha; la escritura y la lectura pasan, entonces, a un segundo plano. Obviamente, se pierde con este razonamiento la consideración del lugar fundamental que ocupa la escritura en nuestra sociedad: nuestra existencia misma está encuadrada -en términos oficiales- por documentos escritos (el acta de nacimiento y el acta de defunción); la escolarización y, con ella, el acceso a la cultura académica y a la posibilidad de ascenso social requieren, ineludiblemente, de saber leer y escribir; la perdurabilidad de lo escrito sigue siendo el factor sobre el cual se basa la administración de la justicia (la ley debe codificarse por escrito), por citar solo algunos ejemplos.

Empero, lo oral, particularmente en su modalidad de conversación, mantiene su lugar de manifestación lingüística primordial: toda persona -salvo muy contadas excepciones- conversa todos los días, independientemente de su nivel de instrucción, su edad, su sexo, su condición socioeconómica o cualquier otro factor; por el contrario, no toda persona escribe todos los días, e incluso muchos individuos alfabetizados raramente leen o escriben. Por lo tanto, pareciera que la escritura sí ocupa un lugar importante en nuestra sociedad, 
pero nunca ha podido desplazar a lo oral de su posición privilegiada como instrumento de comunicación cotidiana, espontánea e inmediata. En este sentido, se espera que quien ha aprendido una lengua sepa manejarla en su versión oral, pero solo como una especie de extra se solicita que sea competente en la versión escrita.

Lo anterior no debe extrañarnos. De hecho, esa ha sido siempre la historia de la humanidad: las lenguas vivas han existido siempre en su oralidad; la escritura es una tecnología sofisticada de creación muy reciente. Las culturas humanas son, en primer lugar, culturas de la oralidad. Solo algunas de ellas -que han logrado imponer en el resto del mundo sus patrones de civilización por encima de los valores y conocimientos autóctonos- llegaron a colocar la escritura en la base misma del ordenamiento jurídico y administrativo de la población.

Que la oralidad es la esencia de las lenguas se comprueba en la gran cantidad de etnias que aún siguen sin escribir sus idiomas o que los han empezado a escribir en fechas recientes por la mediación de instituciones que han diseñado adaptaciones de los alfabetos existentes a las lenguas de esos pueblos; tal es el caso de todas las lenguas indígenas habladas en Costa Rica, las cuales se escriben con alfabeto latino y algunos otros símbolos creados para retratar fenómenos inexistentes en la escritura del español; dichos alfabetos fueron diseñados por lingüistas de la Universidad de Costa Rica con el fin de preservar textos en dichas lenguas y poder registrar los datos que recogían acerca de ellas.

El carácter primario de la oralidad también se hace patente en las lenguas de señas, las cuales se realizan, precisamente, de forma "oral", aunque en ellas no intervenga ningún sonido. Aquí la etimología de la palabra 'oral' pierde toda relevancia (latín os/oris 'boca'), para significar el uso no escrito de la lengua. Por ello mismo, la forma de existencia primordial de las lenguas de señas es la conversación; es decir, la proto-forma más básica de la comunicación humana. Cuando aprendemos LESCO, en este sentido, de lo que se trata es de aprender a conversar en LESCO; la interpretación español-LESCO/LESCO-español es un uso relativamente nuevo de la lengua $\mathrm{y}$, en todo caso, designado a especialistas que deben formarse como intérpretes.

Con toda la argumentación anterior, lo que pretendo sostener es que, para el común de las personas, el uso más primario e importante de las lenguas es su versión oral. Por eso, aprender una segunda lengua es aprender a hablarla en primer lugar. Sin duda esta ha sido siempre la motivación implícita de quienes defienden la oralización de las personas sordas, pues la sociedad espera que sus miembros hablen español.

La competencia verdadera en el nivel escrito importa, sobre todo, en ámbitos intelectuales, por lo que se valora especialmente en términos profesionales y académicos. En nuestra cotidianidad más inmediata, solo se requiere de las personas una alfabetización instrumental, que les permita leer letreros en las calles, números, avisos comerciales y señales de tránsito. No deben interpretarse mis palabras como una apología de lo oral y un desprecio de la cultura escrita, sino únicamente como una constatación de los valores que rigen en nuestra sociedad. En particular, soy un gran defensor de la idea de que nuestra educación debe estar dirigida a lograr niveles muy altos de competencia en el manejo del registro escrito, tanto en los niños y jóvenes oyentes como en los sordos, pero lo que he querido mostrar aquí es la valoración común y diaria que se hace de la escritura frente a la oralidad.

Así, cuando se plantea que el bilingüismo en los sordos reviste una especificidad particular, en el entendido de que una lengua oral (el español, en nuestro caso) no es apta para su aprendizaje en su modalidad oral, por las condiciones acústico-auditivas que tratamos antes, no se debe perder de vista en ningún momento la situación que dibujé en las líneas anteriores. Si bien 
es absolutamente cierto que la adquisición más exitosa del español por parte de una persona sorda es su versión visual (la escrita) y que al desarrollo de la competencia en el código escrito debería estar dirigida la clase de español como segunda lengua, no se debe olvidar que aún persiste el problema de las expectativas de la sociedad. Por ello mismo, no es suficiente que se produzca un cambio en los padres y familiares de las personas sordas, ni tampoco es suficiente que los docentes entiendan bien y puedan aplicar a cabalidad el modelo educativo bilingüe que se propone; mientras para el común de la gente hablar español sea precisamente eso: hablarlo, independientemente de que la persona pueda leerlo y escribirlo a la perfección, la segregación de la persona sorda prevalecerá. En este sentido, cuando se defiende la enseñanza del español como segunda lengua a nivel escrito, en el caso de los individuos sordos, se debe tener presente que con esta decisión se les están proveyendo herramientas para el éxito académico (solo leyendo y escribiendo español puede una persona tener acceso al conocimiento en nuestro sistema educativo), pero no necesariamente para la aceptación social.

De nuevo aclaro que no estoy defendiendo aquí la oralización de los sordos, sino que debe emprenderse la educación de la sociedad oyente para que comprenda que el hecho de que una persona no maneje el español de forma oral no significa que no pueda comunicarse, sino que, en su caso, la forma de transmisión del mensaje descansa sobre lo escrito. En otras palabras, el nuevo modelo de educación bilingüe para la persona sorda debe ir acompañado de una campaña educativa para la población oyente, si es que queremos que dé los frutos esperados.

\subsection{El español escrito}

En cuanto al argumento de que lo prioritario para la persona sorda es el aprendizaje del español escrito, valdría la pena detenerse un poco a reflexionar en lo que ello implica: el registro escrito y el registro oral de la lengua pueden diferir notoriamente, sobre todo si se comparan sus variedades formales e informales.

Ciertamente, las personas sordas reciben en la actualidad un constante estímulo en español escrito por medio de los mensajes de texto del teléfono celular, una forma de comunicación que se ha popularizado entre muchos de ellos y en la población en general; no obstante, no debe olvidarse que tales mensajes se redactan en un estilo muy informal, en el que la ortografía, la puntuación y la precisión no tienen importancia, al igual como sucede en las conversaciones en el chat. Como consecuencia, se trata de una práctica de los usos más informales de la escritura, como ocurre cuando se asignan actividades como llevar un diario de vida o una agenda. Del mismo modo, cuando se dirige la enseñanza a que el niño pueda escribir un recado o solicitar una dirección o hacer una pregunta por escrito en una situación cotidiana, también debe recordarse que estamos ante usos informales de la escritura.

De manera opuesta, leer una noticia o reportaje en el periódico, escribir una redacción escolar o resumir el argumento de un cuento implican usos formales de la escritura. Ambos estilos deben incluirse en la clase, respetando sus características, de modo que resulta inapropiado $-\mathrm{y}$ hasta ridículo- exigir perfecta puntuación en un mensaje de texto o un chat, así como no es conveniente pasar por alto fallas de cohesión y coherencia en una redacción. En pocas palabras, se trata de reconocer lo específico de los diversos usos de la lengua en su manifestación gráfica.

Además, resulta fundamental considerar las diferencias estructurales entre lo escrito y lo oral, en aspectos tan relevantes como el vocabulario y la gramática. Hay estructuras morfosintácticas en español que raramente aparecen cuando hablamos, pero que tienen plena vitalidad en la escritura; tal es el caso del futuro en los verbos 
tipo 'iré', normal al escribir, que en la oralidad cotidiana se reemplaza por 'voy a ir'. Como este, hay decenas de ejemplos en la gramática del español (De Kock y DeMello, 1997). Por lo tanto, habría que estar muy consciente -y muy bien informado- de estas diferencias, con el fin de no caer en generalizaciones y falsedades, como enseñarle a un niño que el futuro que se usa es únicamente 'voy a ir', si se va a encontrar 'iré' tan pronto como lea una noticia o el horóscopo. La identificación y separación claras de las divergencias estructurales y discursivas entre lo escrito y lo oral constituyen un punto básico en la enseñanza del español como segunda lengua; sobre todo, cuando se pretende que el niño sordo desarrolle una buena competencia al escribir para comunicarse tanto en situaciones formales como informales.

En todo caso, la afirmación de que el énfasis debe estar, en el caso de las personas sordas, en la enseñanza escolar de la modalidad escrita del español parte de un hecho indebatible: el canal de transmisión del español escrito coincide, en gran medida, con el canal de realización del LESCO; esto es, ambos basan su recepción y procesamiento en la percepción visual (ver cuadro 1). Si se asume que son los estímulos visuales los que resultan efectivos para las personas con pérdida auditiva severa, se entiende a la perfección por qué es la enseñanza de la versión escrita del español la que tiene mayores posibilidades de éxito y la que resulta más signiticativa e, incluso, más atractiva para el sordo.

\subsection{La lecto-escritura}

Deseo volver nuevamente sobre el papel del LESCO en este modelo educativo bilingüe. Todo apunta a que lo que se pretende es una especie de bilingüismo compartido, en el que el LESCO sea la lengua intragrupal usada en su versión oral, y el español se emplee en su versión escrita como la lengua de comunicación con los oyentes y de acceso a la cultura alfabetizada, además de servir como herramienta de comunicación entre las personas sordas

Cuadro 1

Modelo de relación lengua vocal-lengua de señas (Bouvet, 2000, p. 80)

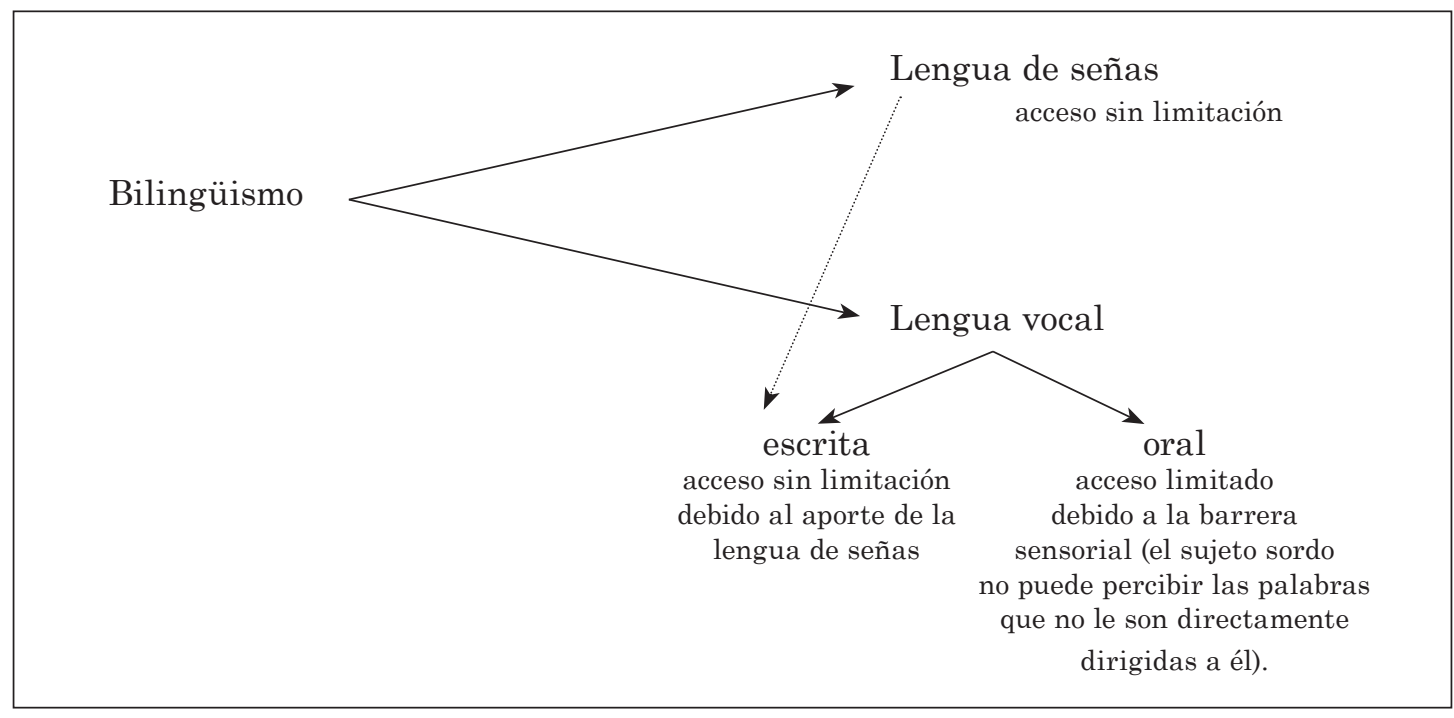


cuando estas no se encuentran cara a cara. Parece lógico que así sea, en vista de que el LESCO carece de un sistema de escritura práctico que les permita a sus usuarios plasmarlo en el papel, digitarlo en la computadora o usarlo para enviar mensajes de texto por teléfono celular. No obstante, este hecho debe llevarnos a una nueva reflexión: dado que el LESCO carece de escritura, en su proceso de alfabetización, los niños sordos deben aprender a escribir en español.

Si se tiene en mente que la escritura del español es de base fonética (sistema fonográfico), surgen nuevas interrogantes; por ejemplo, cómo lograr separar la enseñanza de la lecto-escritura de la realidad fónica -aunque imperfecta- de las letras del español, cómo desligarse de la alusión a la forma en cómo "suena" el símbolo que se está escribiendo. Para un niño sordo que no se oralice, la lógica sobre la que está armada la escritura del español no tiene sentido; el sistema deja de ser económico, aunque esté compuesto de pocos símbolos (unas 26 letras más otros símbolos auxiliares como la tilde y la diéresis), para constituirse en un sistema en el que cada palabra debe aprenderse a escribir de la misma manera en que un niño chino aprende a escribir kanjis, esto es, símbolos que representan unidades mínimas de significado, no unidades de pronunciación.

Cuando aprendemos una nueva palabra en cualquier lengua, debemos aprender $\mathrm{su}$ estructura fonológica (su secuencia de sonidos en una lengua oral, o la secuencia de configuraciones de la mano y su relación con el cuerpo en una lengua de señas). Por ejemplo, aprendemos que la palabra 'casa' en español está conformada por la secuencia fonológica/'kasa/ y que en francés la secuencia es más bien /mezõ/. Esto, inevitablemente, lo aprendemos de memoria, aunque no seamos conscientes de ello. Al aprender a escribir, un niño oyente pasa por un arduo proceso de aprender que esas palabras se pueden descomponer en "pedazos" y que esos pedazos o unidades se pueden representar con signos gráficos, pero tiene la ventaja de que existen pocos signos gráficos, así como existen pocas unidades de pronunciación. Esta es la lógica que sostiene los sistemas de escritura fonográficos: no se representan palabras ni unidades de significado, sino unidades de pronunciación.

Por el contrario, un niño chino, además de aprender la forma oral de la palabra (su combinatoria de fonemas), al alfabetizarse debe aprender un símbolo totalmente diferente para cada palabra; de modo que tendrá que aprender tantos símbolos como palabras haya en la lengua. Esto es así porque el chino desarrolló un sistema de escritura logográfico, en el cual -como se dijo- no se emplean símbolos para representar sonidos, sino unidades de significado; como el chino -al igual que cualquier lengua- posee en su léxico miles de unidades de significado (palabras o morfemas), entonces también tiene miles de signos gráficos para representarlas, no solo unos cuantos símbolos de naturaleza fonética, como el español.

Me parece que, a fin de cuentas, este es también el proceso de alfabetización por el que un niño sordo no oralizado probablemente tenga que pasar: memorizar la secuencia de letras para cada palabra que aprenda en español, del mismo modo en que tendría que memorizar la secuencia de sonidos si estuviera aprendiendo español oral. Aunque parezca requerir un gran esfuerzo, aprender palabras en español escrito de esta manera no difiere mucho de cómo se aprenderían palabras en español oral.

Sin embargo, creo que el aprendizaje de la lecto-escritura en el caso de los niños sordos es mucho más problemático de lo que acabo de plantear. Lo ideal es aprender a escribir en la lengua materna o, en su defecto, en una lengua que a uno le resulte muy familiar, en la que uno tenga una buena competencia o que sea natural para uno. La persona sorda, por su parte, se ve obligada a aprender a escribir en una segunda lengua, la cual, usualmente, ni siquiera ha adquirido en realidad. Es como 
si a cualquier oyente hispanohablante lo obligaran a aprender a escribir en árabe sin saber árabe ni saber escribir en español. Esta situación no pareciera tener una solución pronta ni satisfactoria debido a que el LESCO carece de forma escrita, así que es impensable abordar la enseñanza de la lecto-escritura empezando por el LESCO.

\subsection{El lugar del LESCO en este modelo bilingüe}

Otro aspecto fundamental, en relación con el LESCO, es el deficitario manejo que de él tiene la abrumadora mayoría de los niños sordos que entran a la escuela, si no han tenido la suerte de acceder a los servicios de prematernal que ahora ofrecen algunas instituciones. Como dijimos antes, ¿cómo pretender que el proceso de adquisición del español como segunda lengua sea exitoso si muchos de estos niños en realidad no hablan una primera lengua? Y todavía la interrogante preocupa más cuando tomamos en cuenta que la modalidad de transmisión acústico-auditiva del español impide su adquisición natural en su versión hablada, por lo que se debe aprender en su forma escrita, con el concomitante problema del aprendizaje de la lecto-escritura en el que nos detuvimos antes.

Idealmente, primero debería lograrse el desarrollo fuerte del LESCO como primera lengua, para así poder hablar de la adquisición del español escrito como segundo idioma, en vista de que el español no pareciera prestarse a ser adquirido como primera lengua en el caso de las personas sordas. De no ser de este modo, se estaría enseñando una segunda lengua sin la base de una primera, lo cual es un contrasentido. Debe haber una lengua previa, desarrollada con normalidad, que haya servido de detonante en el proceso de adquisición del lenguaje, que haya surgido paralelamente al desarrollo cognitivo y que, por consiguiente, le sirva de soporte al pensamiento y se preste para alcanzar niveles altos de abstracción lingüística e intelectual. Por ello mismo, forzar a los niños sordos a aprender metalenguaje gramatical (como qué es un 'sustantivo' o una 'oración') en español no solo resulta infructuoso sino hasta -si se quiere- antipedagógico: de hacerse, debería ser primero en LESCO.

Al entrar en el terreno de lo que implica concebir el español como segunda lengua para los sordos y del lugar del LESCO en el nuevo modelo educativo, inmediatamente arribamos a la discusión sobre la educación bilingüe y los diferentes modos de entenderla. Como lo apunta con claridad Peluso:

“(...) los modelos bilingües [en la educación del sujeto sordo] toman en cuenta el lugar de la lengua de señas, en tanto primera lengua, en los procesos de adquisición del lenguaje y del desarrollo del niño. De esta forma introducen la lengua oral y la lengua de señas en la educación de forma independiente una de la otra, promoviendo la interrelación de dos culturas y los procesos de identificación vinculados a la lengua de señas y a la cultura de los sordos" (Peluso, 2000, p. 79).

Lo anterior nos remite, evidentemente, a la pregunta sobre la conveniencia de incluir una clase de LESCO en el currículum para sordos. Este es un punto controversial que suele encender los ánimos. Ciertamente, la respuesta no es sencilla por varios y profundos motivos. En primer lugar, habría que decidir si la clase de LESCO se diseñaría con métodos de enseñanza de la lengua materna o de una segunda lengua.

Volvemos a uno de nuestros puntos iniciales: si se tratara de una lengua materna, el proceso de adquisición para el momento en que los niños entran a la escuela ya debería haberse verificado en su esencia; esto es, el léxico básico ya se habría aprendido, la morfosintaxis de la lengua ya habría alcanzado un gran grado de sofisticación, la fonología estaría desarrollada, y el componente pragmático-discursivo estaría en proceso aún de desarrollo pero con una base ya adquirida (como los patrones de conversación coloquial). Siendo así, la clase de LESCO se ocuparía de desarrollar 
niveles lingüísticos de mayor complejidad, aumentar el vocabulario, practicar estructuras gramaticales poco frecuentes, fomentar la competencia en un mayor número de situaciones comunicativas, enseñar las variedades formales de la lengua, etc.

Sin embargo, si estos niños entran a la escuela sin hablar LESCO, entonces eso significa que apenas a la edad escolar estarán iniciando su proceso de adquisición del lenguaje, con todo lo que ello implica. En tal caso, la clase de LESCO, en alguna medida, tendrá que ocuparse de empezar e incentivar el proceso para que este se dé de forma acelerada, para lo cual podría ser útil recurrir a métodos de enseñanza explícita y controlada de segundas lenguas, además de estímulos lingüísticos de diversa índole.

El que LESCO entre en la escuela responde a varios de los principios contenidos en la Declaración universal de derechos lingüisticos (Artículo 3, inciso 1):

\footnotetext{
"Esta Declaración considera como derechos personales inalienables, ejercibles en cualquier situación, los siguientes: el derecho a ser reconocido como miembro de una comunidad lingüística; el derecho al uso de la lengua en privado y en público; el derecho al uso del propio nombre; el derecho a relacionarse y asociarse con otros miembros de la comunidad lingüística de origen; el derecho a mantener y desarrollar la propia cultura" (Comité de seguimiento, 1996, p. 15).
}

Esta misma Declaración también considera "el derecho a la enseñanza de la propia lengua y cultura" (Artículo 3, inciso 2), así como lo establecido en el artículo 24:

"Toda comunidad lingüística tiene derecho a decidir cuál debe ser el grado de presencia de su lengua, como lengua vehicular y como objeto de estudio, en todos los niveles de la educación dentro de su territorio: preescolar, primario, secundario, técnico, profesional, universitario y formación de adultos" (Comité de seguimiento, 1996, p. 23).

Asimismo, su inclusión como una materia más dentro del currículum (con el nombre, por ejemplo, de "LESCO y cultura sorda") y como el idioma en que se impartirían otras asignaturas respondería a varios de los argumentos que, según Appel y Muysken (1996), se han expuesto a favor de la incorporación de las lenguas minoritarias en la escuela:

a. El progreso académico solo se garantizará si se recurre a la primera lengua del niño como el vehículo inicial de la enseñanza, mientras se aprende la lengua mayoritaria. Si esto no es así, es probable que el fracaso escolar se encuentre a la vuelta de la esquina, como se atestigua en el siguiente relato de Larson (citado por Appel y Muysken, 1996, p. 91), con respecto a los niños amuesa de la selva de Perú: "los niños amuesa comienzan sus estudios sin saber nada o casi nada de español y a veces pasan años antes de que puedan acabar el primer curso porque primero tienen que intentar entender lo que les dice el profesor. La situación generada por el problema comunicativo parecía tan grave que en una de estas escuelas los niños amuesa se encargaban de cuidar el jardín del profesor casi todo el día, mientras que los niños hispano-hablantes recibían clases”. ¿No enfrentan la misma dificultad los niños sordos costarricenses cuando, desde primer grado, deben apreder geografía y matemáticas en español?

b. Si la instrucción no se da en la lengua materna y si esta no se continúa desarrollando en la escuela, entonces los niños verán retrasado su desarrollo cognoscitivo general. Si fuera de la escuela no hay estímulo lingüístico, le corresponde a la institución educativa asumir esta función. Así, es fácil apreciar que la escuela debe estimular a los niños sordos para que desarrollen buena competencia en LESCO, pues el incentivo en el hogar y los barrios de residencia suele ser pobre. 
c. La personalidad del niño y la formación de una autoimagen positiva se ven beneficiadas con la enseñanza en el idioma minoritario. Si la escuela excluye la lengua de los niños, se convierte en un espacio de desconocimiento de su identidad cultural y hasta de rechazo. En este argumento también sobresale el problema de la alfabetización. Está visto que, cuando los niños aprenden a leer y a escribir únicamente en la lengua mayoritaria, desarrollan una noción de desprestigio de su propia lengua, pues asocian el acceso a la educación y el ascenso social con la lengua mayoritaria, y conciben su propia lengua como poco apta y funcional para estos propósitos y, como corolario, la idea de que es una lengua de segunda categoría y ellos son hablantes desprestigiados. Esto nos remite otra vez al problema de la alfabetización del sordo.

d. El desarrollo del lenguaje -como facultad lingüística- requiere del estímulo y desarrollo de la lengua materna del niño, lo cual constituye un prerrequisito para el apredizaje efectivo de la lengua mayoritaria. Al respecto, Appel y Muysken citan la hipótesis del desarrollo interdependiente de Cummins:

\footnotetext{
"Los niños pueden obtener niveles elevados de competencia en la segunda lengua si el desarrollo de su primera lengua, especialmente el uso de determinadas funciones del lenguaje que son básicas para la escolarización y el desarrollo de vocabulario y conceptos, se ve favorecido fuera de la escuela. Un nivel elevado de competencia en la primera lengua hace posible un nivel similar en la segunda. Por otra parte, cuando las habilidades de la primera lengua no están bien desarrolladas y la educación en los primeros años se lleva a cabo sólo en la segunda lengua, el desarrollo posterior de la primera lengua se puede retrasar. Esto provocará, a su vez, un efecto limitador de la adquisición de la segunda lengua (...). Según esta hipótesis, la primera lengua del niño del grupo minoritario se debe desarrollar en la escuela para que la adquisición de la segunda lengua se realice con éxito" (Appel y Muysken 1996, p. 157).
}

Como se ve, sería imperativo, entonces, que el LESCO se desarrolle fuertemente para pensar en la posibilidad de que los niños sordos alcancen siquiera un dominio aceptable del español. Caso contrario, estaremos frente a lo que se ha denominado semilingüismo: el manejo imperfecto de una lengua.

e. Las relaciones socio-culturales entre el grupo minoritario y el grupo mayoritario podrían mejorar si se reconoce la validez de la lengua y la cultura del grupo minoritario. A este respecto, ayuda mucho la incorporación de la traducción simultánea de las noticias al LESCO en algunas de las ediciones de los telenoticieros nacionales.

Sin embargo, un problema, quizás el más delicado, dificulta la inclusión de la clase de LESCO en el currículum: la prácticamente nula descripción de la lengua. Apenas si contamos con una tesis que se ocupa del aspecto verbal (Retana 1993), un artículo de revista dedicado a establecer su origen y relación con otras lenguas de señas (Woodward, 1992) y un diccionario muy básico (López, s.f.). El primer paso para el estudio del LESCO consistiría en la descripción de su estructura en todos los niveles: fonología, morfología, sintaxis, discurso; asimismo, habría que emprender una investigación detallada de los patrones de uso idiomático de la comunidad sorda y de las actitudes hacia su lengua y su posible estandarización con concurso de personas ajenas al grupo.

Debemos estar muy conscientes de que un modelo de educación bilingüe es aquel que contempla la educación en dos lenguas; es decir, no es un sistema bilingüe real aquel en el que la segunda lengua está presente solo en la clase para aprender esta lengua. Por ejemplo, en las escuelas y colegios públicos de Costa Rica se incluyen clases de inglés y francés, pero ello no implica que dicho sistema sea bilingüe, como sí lo sería si la clase de estudios sociales se 
impartiera en español y la de matemáticas en inglés, o si un día la clase de ciencias se dictara en francés y otro día en español.

No voy a entrar en detalle en lo concerniente a los distintos modelos de educación bilingüe, como de si lo que se trata es de utilizar el LESCO en los primeros cursos mientras se adquiere el español para luego abandonarlo (modelo de transición o asimilacionista), o si se va a emplear en todo el proceso educativo al lado del español (modelo pluralista o de mantenimiento). Me parece que esta es una cuestión que debe decidir la comunidad sorda en conjunto con las autoridades del Ministerio de Educación Pública.

Aquí me interesa llamar la atención sobre el hecho de que, dentro de un programa de educación bilingüe para sordos, en el que se pretendan impartir ciertas materias en la lengua de señas, la necesidad de llevar a cabo un proceso de estandarización del LESCO constituye, a todas luces, una tarea fundamental; por ejemplo, es necesario inventariar e incrementar el caudad léxico, especialmente en campos académicos, para poder asegurar la adaptabilidad de la lengua para referirse a distintos campos del saber sin necesidad de tener que recurrir a la constante traducción al español. De no ser así, el modelo tropezará con innumerables escollos para su efectiva implementación, como lo atestigua Peluso (2000) en el caso de Uruguay.

No obstante, para poder emprender tal estandarización se debe tener mucho cuidado de no violentar ni la estructura propia de la lengua ni la sensibilidad de la comunidad sorda con respecto a su medio de comunicación por excelencia, como lo enuncia el Artículo 9 de la Declaración universal de derechos lingüisticos: "Todo comunidad lingüística tiene derecho a codificar, estandarizar, preservar, desarrollar y promover su sistema lingüístico, sin interferencias inducidas o forzadas" (Comité de seguimiento, 1996, p. 19).

Para evitar estos obstáculos, una descripción detallada y sistematizada de la lengua se vuelve indispensable, no solo para la inclusión exitosa del LESCO en el aula de los niños sordos, sino también para la formación de intérpretes y profesores de LESCO, y para la enseñanza de esta lengua a la población oyente interesada. No es conveniente que cada profesor de LESCO enseñe lo que - a su mejor entender y con las mejores intenciones- constituye la lengua, pues se corre el riesgo de estar enseñando un LESCO mitológico que no existe más que en la mente del profesor o en los manuales de gramática de otras lenguas de señas (como el ASL). Se necesitan gramáticas descriptivas y diccionarios que cumplan una función auxiliar en la enseñanza de la lengua.

\subsection{La formación de los docentes}

Llegamos de esta forma al último factor que deseo abordar en este trabajo: la formación de los docentes encargados de los niños sordos. Es esta también una cuestión primordial para el éxito del modelo educativo que se propone: si el maestro no está capacitado en didáctica de segundas lenguas, en la enseñanza del español como idioma segundo y en principios de educación bilingüe, es probable que el programa nunca se llegue a aplicar con efectividad. De ahí la imperiosa necesidad de dotar a los docentes de herramientas teóricas y metodológicas que les permitan enfrentar la educación de las personas sordas en este nuevo enfoque. Debe reconocerse, en consecuencia, la especificidad de la labor de un profesor de segundas lenguas, lo cual es independiente de la formación de los maestros de sordos en educación especial, evaluación, pedagogía y demás disciplinas de su especialidad.

En mi opinión, la formación del docente de sordos debe incluir, en lo que se refiere a su capacitación lingüística, al menos los siguientes aspectos: Adquisición de segundas lenguas; metodología de la didáctica de segundas lenguas; descripción del español en sus diversos componentes: 
fonética-fonología, morfosintaxis, léxicosemántica, análisis del discurso-pragmática; fundamentos de educación bilingüe y bicultural, sociolingüística, dialectología hispánica y psicolingüística; didáctica de la lecto-escritura, con referencia también al estudio lingüístico de los sistemas de escritura; teoría y didáctica del registro escrito, lo que incluye la adquisición del código escrito y la composición; descripción del LESCO en sus diversos niveles; análisis contrastivo LESCO-español.

\section{Reflexión final: El impostergable cambio de actitud en la sociedad}

Aunque pudiera parecer innecesario volver, en este momento, a un asunto tan básico como qué es la sordera, considero que en este caso se justifica, por cuanto este sigue siendo quizás el punto más delicado en lo concerniente a la educación y a la igualdad de oportunidades a la que esta debe aspirar. Defiendo esta posición debido a que nada lograremos mientras siga existiendo el divorcio no solo entre la teoría y la práctica, sino en especial entre lo que se promueve en las aulas y en los círculos académicos y la realidad que viven las personas sordas en la calle. En otras palabras, será tal vez bien poco lo que hayamos logrado si para el común de las personas un sordo sigue siendo un discapacitado, al que hay que hablarle en voz muy alta para que entienda, un ser prácticamente impedido para comunicarse.

Me atrevo a afirmar que, para la mayoría de las personas en nuestro país, incluyendo a muchos padres, la definición de qué es una persona sorda resulta bastante fácil: un sordo es una persona que no oye. Sin embargo, la definición no nos dice nada sobre la persona, sino que más bien enfatiza su condición especial como una discapacidad, una razón para diferenciarla. Tal vez la pregunta misma esté mal planteada (siempre resulta odioso cosificar a las personas con una pregunta del tipo: “¿qué es?"). Deberíamos preguntarnos: ¿quién es una persona sorda? o, mejor aún, ¿qué determina que una persona se considere sorda y qué implica ello?

A este respecto, las palabras de David Crystal son bien reveladoras:

"La dicotomización entre estar sordo (incapaz de responder al sonido) y estar 'no sordo' nunca está clara. Más bien existe un rango potencialmente infinito de grados de pérdida auditiva, que va desde una ligera dificultad para responder a unas cuantas frecuencias de baja intensidad (cosa que apenas interferirá con la comunicación normal) hasta una pérdida donde no existe respuesta detectable a ninguna frecuencia, no importa cuán intenso sea el sonido" (Crystal 1993, p. 168-169).

Es decir, comúnmente englobamos con la etiqueta 'sordo' a un grupo de personas que comparten el rasgo de presentar algún tipo de pérdida auditiva, especialmente cuando esta pérdida es total o casi total. La definición que se puede extraer de las afirmaciones de Crystal me agrada mucho más: una persona sorda es una persona que no responde, en diferentes grados, al sonido, lo cual no tiene nada que ver con su capacidad comunicativa, siempre y cuando se recurra a medios de expresión no dependientes de lo acústico-auditivo: la modalidad escrita del español (o cualquier otra lengua oral) o el LESCO (o cualquier otra lengua de señas).

El cambio en el paradigma educativo formal debe ir acompañado, indefectiblemente, de una campaña educativa nacional, cuyo fin último será que los oyentes comprendan que el LESCO es una lengua al igual que el español, no un sistema de comunicación muy básica compuesto de gestos; es decir, que hablar con una persona sorda implicaría, en gran medida, el mismo comportamiento que asumimos al hablar con un extranjero no hispanohablante que trata de comunicarse con nosotros en español: una actitud tolerante y cooperativa, en la que el mensaje se construye colectivamente gracias al aporte y la participación activa de todos 
los interlocutores, y no una actitud de incomodidad o risa ante el esfuerzo que realiza una persona por expresarse en una lengua que no es la suya.

También habrá que trabajar en la aceptación, por parte del común de la gente, de que las personas sordas constituyen una comunidad lingüística con su propia lengua. Y, claro está, la comprensión de que el LESCO es una lengua, no un lenguaje, ni mímica ni español hablado con las manos; así que aprender LESCO se ubica en la misma posición que aprender inglés o francés en nuestro medio.

En todo este asunto de la conceptualización de quién es sordo, lo que definitivamente queda claro es que el silencio es el gran perdedor (de paso, habrá que erradicar -por falso y prejuicioso- el dicho popular "entrar en un diálogo de sordos", el cual se aplica a situaciones en las que la comunicación no es posible). Se debe entender que las personas sordas no son incapaces de comunicarse ni están en una situación justificada de desventaja. Es por razones de índole social y cultural que estas desventajas surgen. Las personas sordas se comunican perfectamente en LESCO, pero paradójicamente conforman una comunidad de habla sin ubicación geográfica, por lo cual deben vivir inmersos en sociedades con culturas orales y de aquí es de donde nacen las desigualdades. En realidad, de lo que se trata es de entender que una persona sorda recurrirá al español para comunicarse con la comunidad oyente, por ser esta la lengua dominante, pero que utilizará el LESCO con las personas que pertenezcan a su comunidad o con todo aquel que pueda y quiera hacerlo, con independencia de su condición auditiva.

Este cambio de actitud implicará, asimismo, que haya una disposición a comunicarse por escrito, sin reaccionar con extrañeza o desgano. Si este cambio de actitud en la población del país no se suscita, me temo que poco se habrá ganado: los sordos seguirán siendo tratados como deficientes comunicativos, así hablen perfectamente el LESCO y escriban y lean con gran soltura y corrección el español, y -lo que es más grave aún- continuarán siendo segregados y continuarán viendo cómo la comunicación con los oyentes se reduce a una sonrisa fingida y una mirada evasiva.

\section{Notas}

1. Este trabajo se presentó como la primera conferencia magistral del 2005 en el Centro Nacional de Recursos para la Inclusión Educativa.

2. Desde un marco teórico distinto, Peluso (1999) propone características similares para lo que él denomina "lengua materna".

\section{Referencias bibliográficas}

Appel, René y Pieter Muysken. Bilingüismo y contacto de lenguas. Barcelona: Ariel. 1996.

Berko Gleason, Jean y Nan Bernstein Ratner. Psicolingüistica. Madrid: McGrawHill. 1999.

Bouvet, Danielle. "L'accès de l'enfant sourd à la parole: une situation particuliere de bilinguisme entre une langue gestuelle et une langue vocale". En: Estudios de Sociolingüística, Vol. 1, n. 1, pp: 75-81. 2000.

Comité de seguimiento. Declaración universal de derechos lingüísticos. Barcelona: Romanyá Valls S.A. 1996.

Crystal, David. Patología del lenguaje. Madrid: Cátedra. 1993.

De Kock, Josse y George DeMello. Lengua escrita y habla culta en América y España. Salamanca: Ediciones Universidad de Salamanca. 1997. 
Fernández Pérez, Milagros. Introducción a la Lingüistica. Barcelona: Ariel. 1999.

Larsen-Freeman, Diane y Michael H. Long. Introducción al estudio de la adquisición de segundas lenguas. Madrid: Gredos. 1994.

López Grazioso, Diana. s.f. Comuniquémonos mejor. (s.e.)

Peluso Crespi, Leonardo. "Reflexiones acerca de la lengua materna, la sordera, la fonoaudiología y algunas cosas más”. XII Congreso Internacional de ALFAL, Santiago de Chile. 1999.

Peluso Crespi, Leonardo. "Políticas educativas y lingüísticas en la educación del sordo: Hacia una educación culturalmente sensible". En: Peluso y Torres, pp. 79-94. 2000.

Peluso, L. y C. Torres. Indagaciones en los márgenes. Cuestiones sobre lenguaje, psicología y educación. Montevideo: Ed. Trilce. 2000.

Retana Castro, Priscilla. Descripción del aspecto verbal en la lengua costarricense de señas. Tesis de Maestría, Universidad de Costa Rica. 1993.

Sampson, Geoffrey. Sistemas de escritura. Análisis lingüístico. Barcelona: Gedisa. 1997.

Sánchez López, M ${ }^{\mathrm{a}}$ Pilar y Rosario Rodríguez de Tembleque. El bilingüismo. Bases para la intervención psicológica. Madrid: Síntesis. 1997.

Vega, Virginia. "Factores psicológicos que intervienen en el aprendizaje de lenguas extranjeras". Revista de Filología y Lingüística XVI (1): 139148. 1990.

Veyrat Rigat, Montserrat. Bilingüismo, traducción e interpretación de lenguas. Aplicación al lenguaje de signos. Valencia: Centro de Estudios sobre Comunicación Interlingüística e Intercultural. 1998.

Woodward, James. "Historical Bases of New Costa Rican Sign Language", Revista de Filología y Lingüistica XVIII(1): 127-132. 1992. 